\title{
The Involvement of Neuroinflammation and Kynurenine Pathway in Parkinson's Disease
}

\author{
Anna Zinger, ${ }^{1}$ Carlos Barcia, ${ }^{2}$ Maria Trinidad Herrero, ${ }^{2}$ and Gilles J. Guillemin ${ }^{1,2,3}$ \\ ${ }^{1}$ Department of Pharmacology, School of Medical Sciences, University of New South Wales, Sydney, NSW 2052, Australia \\ ${ }^{2}$ Experimental and Clinical Neuroscience (NiCE-CIBERNED), Department of Human Anatomy and Psychobiology, \\ School of Medicine, University of Murcia, Murcia, Spain \\ ${ }^{3}$ St Vincent's Centre for Applied Medical Research, Darlinghurst, NSW 2010, Australia
}

Correspondence should be addressed to Gilles J. Guillemin, g.guillemin@unsw.edu.au

Received 1 December 2010; Accepted 31 January 2011

Academic Editor: Heinz Reichmann

Copyright ( $) 2011$ Anna Zinger et al. This is an open access article distributed under the Creative Commons Attribution License, which permits unrestricted use, distribution, and reproduction in any medium, provided the original work is properly cited.

Parkinson's disease (PD) is a common neurodegenerative disorder characterised by loss of dopaminergic neurons and localized neuroinflammation occurring in the midbrain several years before the actual onset of symptoms. Activated microglia themselves release a large number of inflammatory mediators thus perpetuating neuroinflammation and neurotoxicity. The Kynurenine pathway (KP), the main catabolic pathway for tryptophan, is one of the major regulators of the immune response and may also be implicated in the inflammatory response in parkinsonism. The KP generates several neuroactive compounds and therefore has either a neurotoxic or neuroprotective effect. Several of these molecules produced by microglia can activate the N-methylD-aspartate (NMDA) receptor-signalling pathway, leading to an excitotoxic response. Previous studies have shown that NMDA antagonists can ease symptoms and exert a neuroprotective effect in PD both in vivo and in vitro. There are to date several lines of evidence linking some of the KP intermediates and the neuropathogenesis of PD. Moreover, it is likely that pharmacological modulation of the KP will represent a new therapeutic strategy for PD.

\section{Introduction}

Parkinson's disease (PD) is the most common movement disorder and is the second most common chronic progressive neurodegenerative disorder after Alzheimer's disease. PD is a sporadic and age-dependent disease in $90 \%$ of cases and affects more than $1 \%$ of the world population over the age of 65 [1]. PD is characterised by motor symptoms including bradykinesia, tremor, rigidity, postural instability as well as nonmotor symptoms such as dementia, sleep disturbance, neurobehavioral, and sensory abnormalities [2].

PD is neuropathologically characterized by the loss of midbrain-pigmented neurons in the substantia nigra pars compacta (SNpc). Under normal conditions, these neurons produce dopamine at the striatum and other basal ganglia nuclei [3]. It has been estimated that at the onset of PD symptoms, up to $70 \%$ of dopaminergic neurons have been lost. Postmortem examinations have also shown that more than $90 \%$ of these neurons have been depleted [4]. Dopaminergic loss leads to an irreversible degeneration of the nigrostriatal pathway, followed by stratial dopaminergic denervation which causes pathological changes in neurotransmission of basal ganglia motor circuit and results in characteristic Parkinsonian symptoms [5]. Another pathological hallmark of the disease is the presence of protein inclusions called Lewy bodies (LBs), which are abnormal intracellular $\alpha$ synuclein (SYN) aggregates in the cytoplasm and axons of the remaining neurons [6]. Neurons containing LBs undergo neurodegenerative processes and subsequently die.

To date, there is no available cure for PD. However, L-Dopa and dopaminergic agonists are useful in treating PD symptoms. This type of therapy mainly aims to replace dopamine in the striatum but does not slow neurodegenerative processes. Moreover, long-term use is associated with serious side effects such as dyskinesia and motor fluctuations [7] resulting in a diminished effect of treatment [8].

Although the aetiology of PD is relatively unknown, it has been suggested that there is an association with mitochondrial dysfunction in nigral neurons and neurotoxicity from excess glutamate and reactive oxygen species (ROS) 
production $[9,10]$. Microglia are the prime immune cells of the central nervous system (CNS) and are important producers of neuroactive molecules involved in oxidative stress, excitotoxicity and neuroinflammation. Microglia respond to a wide range of immunologic stimuli or CNS injuries and either initiate protective and/or neuroinflammatory processes [11]. The SN contains the highest concentration of microglia compared with other brain areas [12].

Resting microglia have a characteristic ramified morphology; the small cell body remains stationary whilst the long branches are constantly moving and are sensitive to any minor physiological changes $[13,14]$. At the site of inflammation, activated microglia change their morphology becoming amoeboid and may act similarly to macrophages: they possibly perform phagocytosis, express increased levels of major histocompatibility complex (MHC) antigens, and secrete various cytotoxins, which may ultimately activate additional microglia to remove harmful stimuli and even initiate healing processes $[15,16]$. The total number of MHC class II microglia has been shown to be significantly increased not only in SN and putamen but also in the hippocampus, transentorhinal cortex, cingulate cortex, and temporal cortex in PD brains [17]. This implies that microglia are activated and are likely to be associated with the neuropathological phenomenon, which ultimately damages neurons $[17,18]$. The microglial reaction is a very tightly regulated process which is essential for a precise immune response; excessive microglial activation leads to a continuous release of inflammatory mediators such as cytokines, chemokines, reactive free radicals, and proteases [19]. This process is referred to as "reactive microgliosis" and involves the proliferation, recruitment, and activation of microglia which is then followed by neuronal damage [20], all of which are secondary to actual neuronal injury. Thus, initial, acute damage from microgliosis may provoke a continuous cycle of events, which then develops into chronic, progressive neurodegeneration which is a common characteristic of Parkinson's disease [21].

\section{The Role of Neuroinflammation in the Pathogenesis of PD}

A large number of studies involving cells, animal models, and human patients indicate the involvement of neuroinflammation in the neuropathogenesis of PD.

2.1. In Vitro/In Vivo. To demonstrate the delayed and progressive nature of neuroinflammation observed in PD, lipopolysaccharide (LPS) was administered to rodents as a single dose or a chronic infusion [22]. While LPS has no direct effect on neurons, it is capable of initiating a chronic inflammation and a delayed, secondary progressive degeneration of dopaminergic neurons in the $\mathrm{SN}[22,23]$. An in vitro study has also shown that 1-methyl-4-phenyl-1,2,3,6-tetrahydropyridine (MPTP) can initiate direct neuronal injury in neuron-glia cultures which is then followed by the induction of reactive microgliosis [24]. Furthermore, in a microglia free neuronal-astrocytic coculture, MPTP induced only acute, non-progressive neurotoxicity [21]. MPTP is selectively toxic to dopaminergic neurons and is often used to induce an in vivo PD-like disease in animal models [25]. Moreover, inhibition of microglial activation results in a strong decrease in neurotoxicity in both MPTP mouse and LPS rat models $[26,27]$.

2.2. Human Studies. A large epidemiological study on approximately 150,000 men and women has shown that the use of nonsteroidal anti-inflammatory drugs (NSAIDs) can prevent or delay the onset of PD [28]. Chen et al. have also observed a similar effect in chronic users of ibuprofen, a NSAID acting on cyclooxygenase (COX) [29]. A correlation has also been found between high plasma concentrations of interleukin-6, a proinflammatory cytokine, and an increased risk of developing PD [30]. Moreover, in vivo imaging studies on patients with idiopathic PD have shown an increase in neuroinflammatory areas in basal ganglia, striatum, and frontal and temporal cortical regions compared with agematched healthy controls [31]. All of these studies suggest that microglial activation occurs at an early stage of the disease either before (or in parallel with) the important loss of dopaminergic neurons. In postmortem PD tissues, activated microglial cells have been detected around impaired dopaminergic neurons in the $\mathrm{SN}$, thus demonstrating the presence of neuroinflammation [32]. As previously discussed, MPTP causes Parkinsonism in both humans and primates. This leads to the chronic presence of activated microglia around dopaminergic neurons in the SN for up to 10 years after exposure $[33,34]$, even without L-DOPA treatment [35]. Substantial evidence of microglial activation associated with dopaminergic neuronal damage suggests that degenerating neurons initiate microgliosis, which then leads to further neuronal loss. Microglial activation represents an initiator and/or a secondary responder in this disease process. Therefore, suppressing neuroinflammation by preventing microglial activation could potentially slow down or stop this continuous and deleterious cycle which damages neurons.

However, the initial stimulus driving excessive inflammation is still unknown. There are several compounds released by damaged neurons, which are able to induce microgliosis and ROS production. These include (i) matrix metalloproteinase 3 (released by damaged dopaminergic neurons), which induces superoxide production by microglia leading to neuronal death [36]. (ii) Neuromelanin, a neuronal pigment released in PD by dying neurons which is capable of activating microglia [37]. (iii) SYN, a component of LB neurons, typically found in PD that is toxic to neurons but only in the presence of microglia. (iv) Aggregated SYN-activated microglia are toxic to dopaminergic neurons isolated from embryonic mouse brain. Importantly, its toxicity is dependant on the presence of nicotinamide adenine dinucleotide phosphate (NADPH) oxidase following ROS formation [38]. Another study has shown that neuroinflammation is accompanied by dopaminergic loss and aggregation of oxidized SYN in the cytoplasm of SN neurons when human SYN is present in the mouse brain [39]. Taken together, these studies suggest that there is a link between protein aggregation and the production of ROS by activated microglia. 
Over production of ROS by microglia has been directly linked to neuronal toxicity and death via the nitric oxide (NO) mechanism $[40,41]$. NO induces oxidative stress, a major cause of neuronal injury, which is strongly linked to the pathogenesis of PD and physiological aging [42, 43]. For example, NO can react with dopamine to generate quinone products, which are known to have a damaging effect on brain mitochondria [44]. Basal level of lipid peroxidation is increased in the SN of PD patients, suggesting a higher sensitivity of this area to free radicals and ROS [45]. Aging also contributes to microglial "priming": activated microglia in healthy aged brains release excessive quantities of proinflammatory cytokines compared to younger individuals $[46,47]$. Furthermore, there is an increased probability of developing a neurodegenerative disorder after 60 years of age due to age-related increases in oxidative, metabolic, or inflammatory activation [48].

Inflammatory cytokines (IL- $1 \beta$, TNF- $\alpha$, IL-6, and IFN$\gamma$ ) are also released by activated microglia and amplify the inflammatory response. Excessive production of these cytokines has been reported in the SN of PD patients $[49,50]$ as well as in cerebrospinal fluid (CSF) and blood compartments $[51,52]$. Cytokines can stimulate inactivated microglia and also directly bind to receptors on the cellular surface of dopaminergic neurons thereby promoting apoptotic cell death and subsequent phagocytosis of DA neurons [53]. Neurons in the midbrain, unlike those in the hippocampus or cortex, exhibit a greater sensitivity to proinflammatory cytokines. Moreover, this sensitivity has been directly related to a high degree of oxidative processes [19]. In contrast, activated microglia also produce anti-inflammatory cytokines such as TGF- $\beta 1$, IL-10, and IL-1. These cytokines play a role in the inhibition of the inflammatory response. Importantly, the balance between pro- and anti-inflammatory cytokine production is impaired during neuroinflammation [54].

On the other hand, the excitatory neurotransmitter glutamate plays a critical role in glutamatergic transmission in basal ganglia functions [55]. The action of glutamate on neurons is mediated by ionotropic and metabotropic glutamate receptors. Ionotropic N-methyl-D-aspartate (NMDA) receptors are known to mediate excitotoxicity caused by high levels of glutamate and can be found on dopaminergic neurons [56]. Activation of NMDA receptors located on DA neurons leads to neurotoxicity both in vitro and in vivo $[57,58]$. The functional organisation of basal ganglia also contributes to the genesis of symptoms observed in movement disorder. The striatum (the input nucleus of the basal ganglia circuit) is the main recipient of dopaminergic fibres from the $\mathrm{SN}$. The reduction in dopaminergic innervations of the striatum and changes in the activity of basal ganglia induces complex changes in the structure and function of basal ganglia NMDA receptor [59]. Glutamatergic excitation is increased and glutamatergic neurons become uninhibited under PD conditions, especially due to the excessive firing from the subthalamic nucleus to the SN [60] (Figure 1). It has been shown that the neurotoxicity of activated microglia is primarily mediated by glutamate released through NMDA receptor signalling [61]. Neuritic beading (a focal beadlike swelling in dendrites and axons) is a neuropathological

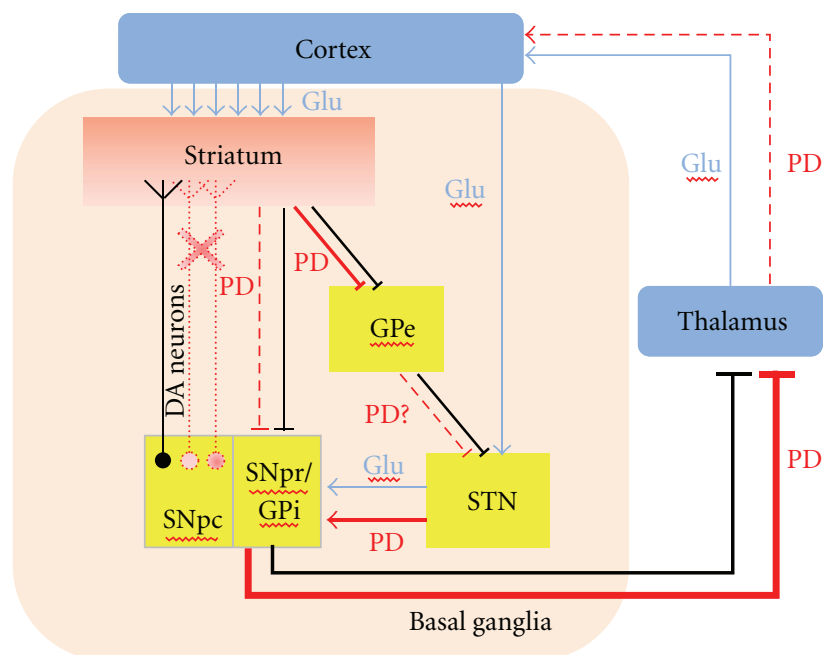

Figure 1: Basal ganglia motor circuit in Parkinson's disease: dopaminergic neurons (DA) create a direct pathway between Substantia Nigra pars compacta (SNpc) and striatum-the input nuclei of the basal ganglia. Another direct pathway connects the striatum to the internal segment of globus pallidus (GPi) and the substantia nigra pars reticulata (SNpr). GPi and SNpr are the output nuclei of the basal ganglia, which projects to the thalamus and from there to the cortex. The indirect pathway connects the striatum to output nuclei through external segment of the globus pallidus (GPe) and then subthalamic nucleus (STN). In Parkinson's disease (PD), the dopaminergic input from $\mathrm{SNpc}$ is progressively lost, causing a reduction in the direct pathway signal. Indirect pathway increases its activity through STN in the output nuclei and has inhibitory influence on the thalamus. It leads to a reduction of thalamic glutamateric input on the motor cortex and subsequent reduction in movement, as rigidity and bradykinesia are observed in PD patients.

sign in PD [62]. It can also be induced by microglia activated through the NMDA receptor [61]. NMDA receptors have been linked with disturbed energy metabolism and glutamate transmission leading to neuronal death, and have therefore been investigated as important therapeutic targets in pharmacological PD research [63]. Accordingly, reducing glutamatergic transmission may lead to an "antiPD activity". Indeed, injections of the NMDA antagonist, MK-801, reverses parkinsonian symptoms in MPTP-treated monkeys [64]. Several studies using rodent PD models have shown that glutamate antagonists have both symptomatic and neuroprotective effects in PD [59]. Recently, PD patients treated with memantine, another NMDA receptor antagonist have shown moderate but significant improvements in terms of cognitive symptoms [65]. The use of amantadine as an adjuvant to levodopa has demonstrated beneficial effects on motor response complications [66]. Additional evidence has been reviewed and has demonstrated the potential of NMDA receptor blockade in reversing parkinsonian symptoms [59].

\section{The Kynurenine Pathway}

The kynurenine pathway (KP) represents the main catabolic pathway of the essential amino acid tryptophan (TRP), 


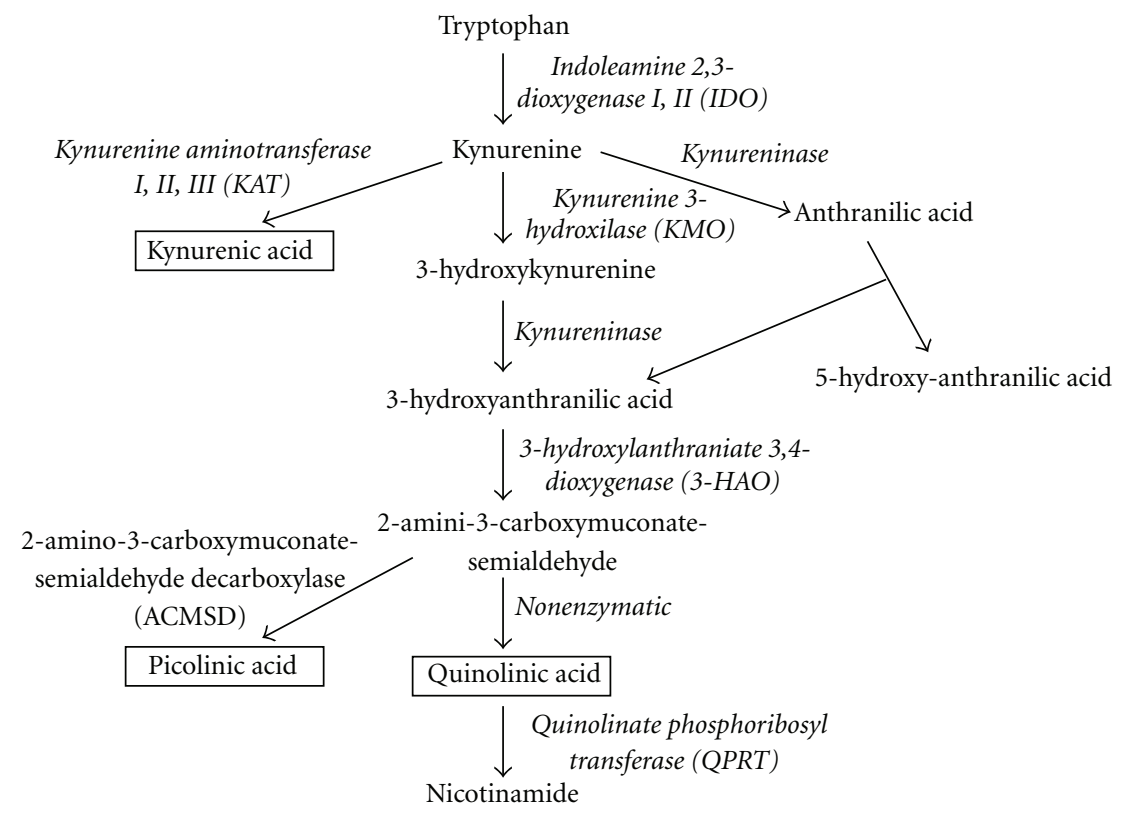

FIGURE 2: Simplified diagram of Kynurenine pathway: during neuroinflammation, 95\% of the dietary tryptophan is metabolized along the $\mathrm{KP}$ within the brain. The remaining 5\% serves as a precursor to the synthesis of the neurotransmitter serotonin. IDO catalyses the initial and rate-limiting step in the degradation of tryptophan through KP that ultimately leads to the production of nicotinamide.

which ultimately leads to the production of the central metabolic cofactor, nicotinamide adenine dinucleotide $\left(\mathrm{NAD}^{+}\right.$) (Figure 2). The KP is also one of the major regulatory mechanisms of the immune response [67]. Two nonmutually exclusive theories have been proposed: (1) that TRP degradation suppresses T-cell proliferation by dramatically depleting the supply of this critical amino acid and (2) that various downstream KP metabolites suppress certain immune cells [67]. Induction of the KP by the ratelimiting enzyme, indoleamine 2,3 dioxygenase (IDO1) in dendritic cells completely inhibits clonal expansion of $\mathrm{T}$ cells [68]. Moreover, TRP depletion and IDO1/KP activation have been implicated in the facilitation of immune tolerance associated with pregnancy and tumour persistence [69].

The cellular expression of the KP in the brain is only partially understood. It is complete in cells of monocytic lineage, including macrophages and microglia [70], but only partially present in human astrocytes [71], neurons [72], and endothelial cells [73]. The various KP metabolites can have either neurotoxic or neuroprotective effects and occasionally both depending on their concentration. The neurotoxicity of several KP metabolites has been investigated in relation to oxidative stress generation and neuronal death in vitro and in vivo in animal models of neurodegenerative disorders [74-77]. 3-hydroxykynurenine (3-HK), 3hydroxyanthranilic acid (3HAA) and 5-hydroxyanthranilic acid (5HAA) are known to induce cell death in cultures of rat neurons [78]. 3-HK is toxic to stratial neuronal cultures, mainly due to its ability to generate ROS and initiate apoptosis [79]. Quinolinic acid (QUIN) however, is likely to be the most important in terms of biological activity. QUIN can selectively activate NMDA receptors producing excitation and which ultimately causes selective neuronal lesions in the rat brain $[80,81]$. Acute QUIN production can lead to human neuronal death and chronic production causes dysfunction by at least six separate mechanisms $[82,83]$. In pathophysiological concentrations, QUIN activates the NMDA receptor [84]. QUIN also increases glutamate release in neurons and inhibits glutamate uptake and catabolism in astrocytes. QUIN can potentiate its own toxicity and that of other excitotoxins, for example, NMDA and glutamate thus producing progressive mitochondrial dysfunction [85]. Finally, QUIN can increase free radical generation by inducing nitric oxide synthase production (NOS) in astrocytes and neurons which in turn leads to oxidative stress $[86,87]$. Within the brain, QUIN is produced by activated microglia and infiltrating macrophages [70]. Neurons and astrocytes do not produce QUIN [88, 89]. Recent findings have demonstrated that QUIN excitotoxicity in human astrocytes and neurons is mediated through activation of an NMDA-like receptor [87]. In addition, QUIN-induced damage can be increased in the presence of 3-HK, 6-hydroxidopamine, a specific dopaminergic neuron toxin, or ROS [90-92]. Human glial cells, such as astrocytes and microglia produce most components of the KP [93]. The KP components are also present in macrophages that are capable of penetrating the blood-brain barrier (BBB) in the presence of brain damage or infection [94]. Thus, up-regulation of QUIN production alone or with additional neurotoxic factors during inflammation could easily lead to over activation of the NMDA receptor. This is followed by oxidative stress, which occurs in early PD development.

In contrast to the neurotoxic activity of QUIN, kynurenic acid (KYNA) is a neuroprotective metabolite, antagonising 
all ionotropic glutamate receptors (including NMDA) and thus blocks some of the neurotoxic effects of QUIN and other excitotoxins. KYNA is produced from kynurenine by the kynurenine aminotransferase enzymes (KAT) I, KAT II, and KAT III, in astrocytes [71]. Endogenous generation of KYNA in rat brain has been shown to be more effective than KYNA applied exogenously, suggesting the importance of localised KYNA production and physical proximity to NMDA receptors [95]. An increase in endogenous KYNA levels can prevent SN dopaminergic loss caused by focal infusion of QUIN or NMDA [96]. Nanomolar concentrations of KYNA significantly reduce glutamate output from striatal neurons in rat brain, similar to the kynurenine hydroxylase (KMO) inhibitors [97]. Both, KYNA and QUIN are produced in the $\mathrm{SN}$ or the adjoining striatum region $[98,99]$. Based on previous studies, it can be hypothesised that under normal conditions local concentrations of KYNA and QUIN are low and physiologically regulate NMDA receptor function. However, in disease states, where QUIN production is high, it is thought that there is insufficient KYNA concentration to block QUIN production [100].

Picolinic acid is another endogenous neuroprotective compound [101] and is also the main metal chelator in the brain [102]. Previously, we have shown that it is produced in micromolar concentrations by human primary neurons [72]. $\mathrm{PD}$ is associated with neuropathological features such as protein aggregation and oxidative stress associated with the involvement of metal ions [103]. Therefore, use of chelating agents has also been suggested as a form of therapy for PD.

The KP, under normal physiological conditions is well balanced and produces all KP intermediates leading ultimately to $\mathrm{NAD}^{+}$production. However, under pathologic conditions, IDO1 is activated and astrocytes produce kynurenine (KYN) and KYNA, [104], neurons produce PIC [88] and activated microglia/infiltrating macrophages produce QUIN [89]. It is important to note that PIC and KYNA can partly antagonise the neurotoxic effects of QUIN [105]. However, astroglial secretion of large quantities of KYN can lead to further synthesis of QUIN by microglia, suggesting that the cerebral synthesis of QUIN largely overtakes the neuroprotective effects of PIC and KYNA [106].

\section{Evidence for the Involvement of the KP in PD}

Impaired KP metabolism and altered KYNA levels have been previously reported in the brain of $\mathrm{PD}$ patients. This occurs when the KYNA/TRP ratio in serum and cerebrospinal fluid (CSF) is significantly increased together with 3-HK levels, a neurotoxic compound that contributes to oxidative damage in the putamen and SNpc $[107,108]$. These findings suggest that endogenous KYNA concentrations are decreased and unable to effectively block NMDA receptor and prevent neurotoxicity induced by 3 -HK. KAT I expression, the KP enzyme which leads to KYNA formation, is decreased in the SNpc of MPTP-treated mice [109]. KAT-I immunoreactivity in dopaminergic neurons and surrounding microglia has been linked to increased vulnerability of SN neurons to toxicity. Lowered KYNA concentrations have also been found
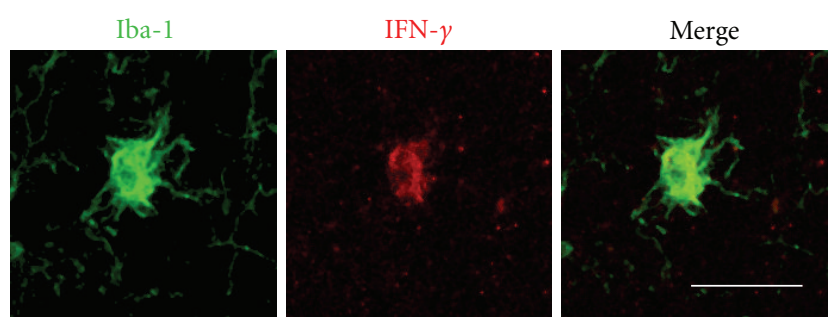

FIGURE 3: Activated microglial cells express IFN- $\gamma$ in Parkinsonism: confocal images of the immunofluorescence of IFN- $\gamma$ (red) combined with microglia cells marker-Iba-1 (green) in the SNpc of a parkinsonian monkey. Scale bar: $35 \mathrm{~mm}$.

in the frontal cortex, putamen, and SNpc of PD patients [107]. KYNA, but not the highly selective NMDA antagonist 7-chlorokynurenic acid exhibits partial protection against $\mathrm{MPP}+$ toxin on dopaminergic terminals of rat striatum [110].

However, increased KAT II activity, which is an enzyme responsible for $75 \%$ of the KYNA synthesis in the brain, has been found in peripheral red blood cells of PD patients. It is not however found in plasma [111]. The increased KAT II activity correlates with higher blood KYNA concentrations; this elevation may be caused by 3 -HK released from the CNS. As KYNA has limited abilities to cross the BBB, it has been suggested that peripheral KYNA is likely to be transported to the brain by large neutral amino acid carriers and there it has neuroprotective effects [112]. Another recent study has shown that KYNA is involved in leukocyte recruitment and the investigators hypothesised that KYNA might therefore have an anti-inflammatory action [113]. Based on preclinical and clinical data, KYNA or its analogues are thought to have neuroprotective effects in PD trough binding as antagonists to the NMDA receptor. This in turn causes slow neuronal excitotoxic damage [114].

Unpublished data from our group shows an increase in the production of IFN $-\gamma$ by microglia in the SN of MPTPtreated macaques' brain (Figure 3). This is of particular significance, as IFN- $\gamma$ is also a potent inducer of the KP [115]. In the same study, we have also shown that QUIN is produced and accumulated by activated microglia. These microglia colocalise with dopaminergic neurons in the SN of MPTP-treated macaques. Several other studies have shown extensive evidence of activated microglial cells and $\mathrm{NMDAR}^{+}$dopaminergic neurons in the SNpc. This suggests that the NMDA receptor is likely to be activated by endogenous QUIN released by microglia and followed closely by glutamate [116, 117] (Figure 4).

\section{Recent KP Inhibitors for the Treatment of PD}

Several drugs that block the KP are currently under therapeutic investigation both in our laboratory and by other investigators. For example, 4-chlorokynurenine crosses the $\mathrm{BBB}$ and blocks QUIN toxicity at the glycine site on NMDA receptors [118]. Kynurenic acid analogues are currently due to enter clinical trials for the treatment of epilepsy, 


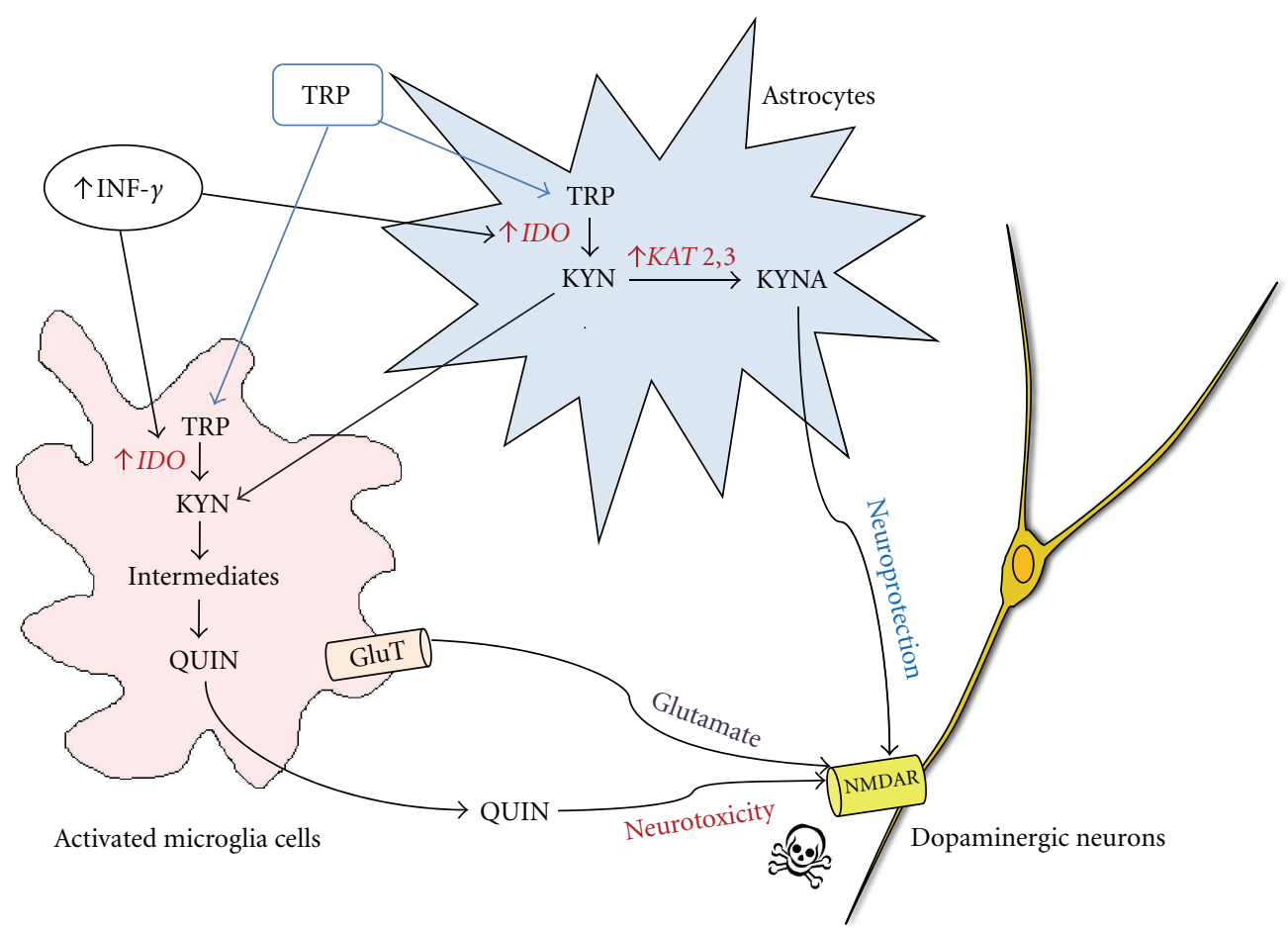

FIGURE 4: Model for Kynurenine pathway interactions between astrocytes, neurons, and microglia during brain inflammation. Abbreviations: TRP: tryptophan; IDO: Indoleamine 2,3-dioxygenase; KYN: kynurenine: QUIN: quinolinic acid; NMDAR: NMDA receptor; KAT: Kynurenine aminotransferase; GluT: glutamate transporter.

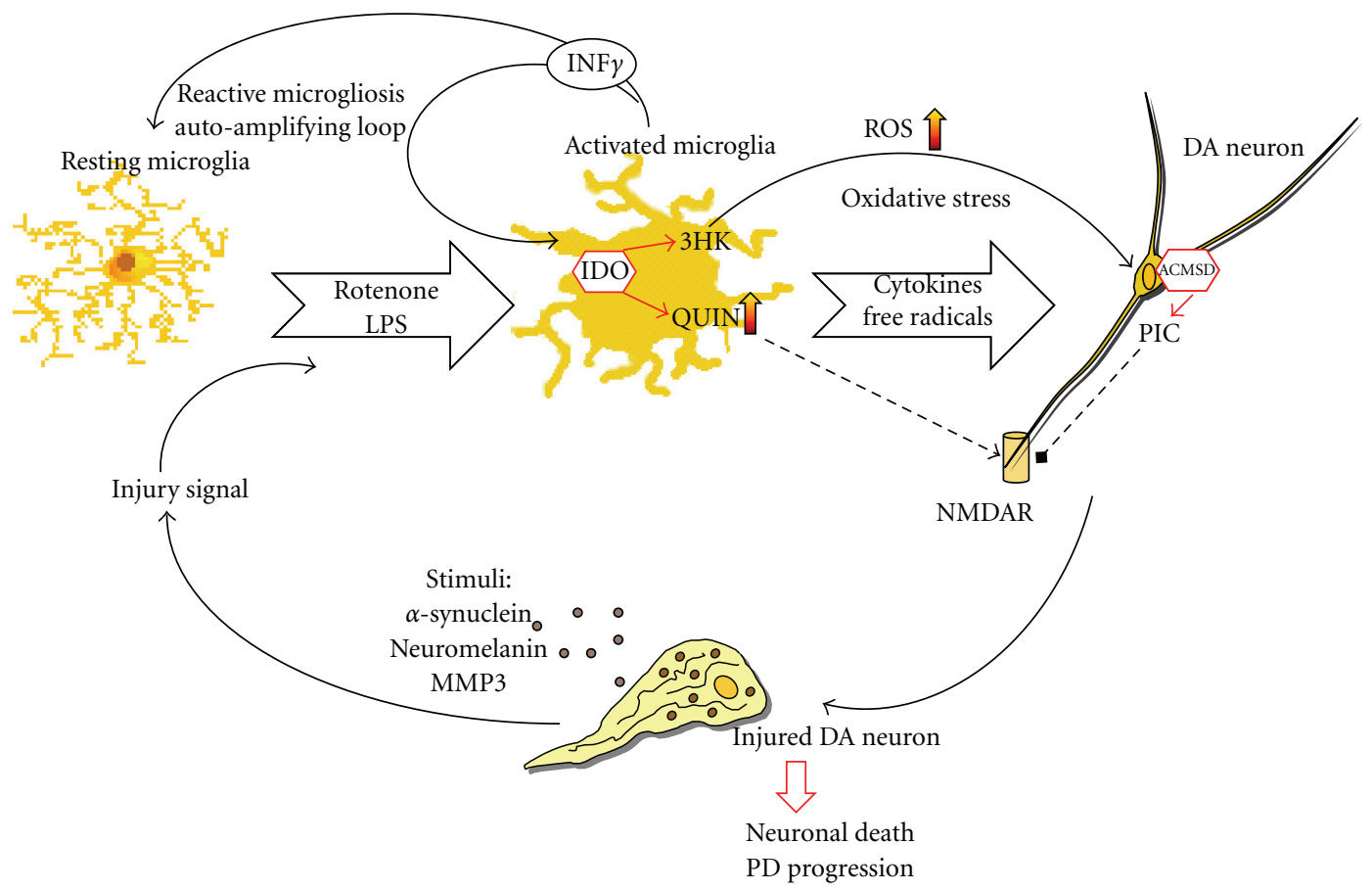

FIgURE 5: The possible role of Kynurenine pathway involvement in dopaminergic neurodegenerative process through microglia activation: Parkinson's disease is associated with chronic activation of microglia, which also can be induced by LPS or Rotenone treatments. Classic microglia activation release neurotoxic substances including reactive oxygen species (ROS) and proinflammatory cytokines as INF- $\gamma$, potent activator of Kynurenine pathway (KP). KP in activated microglia leads to upregulation of $3 \mathrm{HK}$ and QUIN. 3HK is toxic primarily as a result of conversion to ROS. The combined effects of ROS and NMDA receptor-mediated excitotoxicity by QUIN contribute to the dysfunction of neurons and their death. However, picolinic acid (PIC) produced through KP activation in neurons, has the ability to protect neurons against QUIN-induced neurotoxicity, being NMDA agonist. Microglia can become overactivated, by proinflammatory mediators and stimuli from dying neurons and cause perpetuating cycle of further microglia activation microgliosis. Excessive microgliosis will cause neurotoxicity to neighbouring neurons and resulting in neuronal death, contributing to progression of Parkinson's disease. 
stroke, and possibly PD as potential neuroprotective agents [119]. Two KP analogues are at present under investigation in a phase III clinical trial. These are Teriflunomide (Sanofi-Aventis) and Laquinimod (Teva Neuroscience) [120]. Recently, one KP analogue reached the Japanese market as a potent immunomodulatory drug for the treatment of arthritis, asthma, and dermatitis [120]: Tranilast/Rizaben (Kissei Pharmaceutics) is an anthranilic acid derivative and it has been proposed as a treatment for autoimmune diseases such as Multiple Sclerosis [121]. Finally, 8-OH Quinolinone metal attenuating compounds-Clioquinol and PBT2 (Prana) rapidly decrease soluble brain amyloid-beta and improve cognitive performance [122]. Interestingly, these 2 compounds share close structural similarity and similar biochemical properties with KYNA and QUIN.

Conjugates of KYNA analogues with D-glucose or Dgalactose increase its ability to cross the $\mathrm{BBB}$ and prevent excitotoxicity and seizures in an animal model [123]. Kynurenine 3-hydroxylase inhibitors significantly reduce the severity of dystonia in hamsters and may therefore be a potential candidate for managing dyskinesia associated with striatal dysfunction [124]. There is also an increasing interest in the use of pharmacological modulation of the $\mathrm{KP}$ in treating numerous disorders like AIDS-dementia and many other neurodegenerative diseases, diabetes, depression, infections, tumour development, glaucoma, and cataract formation [116].

\section{Conclusions}

PD seems to be associated with an imbalance between the two main branches of the KP within the brain. KYNA synthesis by astrocytes is decreased and concomitantly, QUIN production by microglia is increased (Figure 5). There are many therapeutic opportunities for intervention and modification of an impaired KP that may prevent the progression of neurodegenerative disorders such as PD. Using specific KP enzyme inhibitors, it may be possible to reinstate a physiologically normal KP, which is neuroprotective. This neuroprotective state might also be synergistically improved by concomitantly blocking the NMDA receptor using its antagonists, such as memantine or MK801. Additionally, neuroprotection may be achieved by designing KYNA analogues that are able to penetrate the $\mathrm{BBB}$ and deliver neuroprotective compounds to brain pools thus reducing hyperactivation of glutamatergic receptors.

\section{Acknowledgments}

The authors thank Nady Braidy, Seray Adams, and Louise Pemberton for their critical reading and comments on this paper. They also thank Parkinson NSW for supporting their research on PD. This work was also supported by grants from: the Spanish Ministry of Science and Innovation (FIS PI10 02827, SAF2010-2127, and RYC-2010-06729), Fundación Séneca (15329/PI/10), CIBERNED (Centro de Investigación Biomédica en Red sobre Enfermedades Neurodegenerativas).

\section{References}

[1] C. M. Tanner, "Epidemiology of Parkinson's disease," Neurologic Clinics, vol. 10, no. 2, pp. 317-329, 1992.

[2] J. Jankovic, "Parkinson's disease: clinical features and diagnosis," Journal of Neurology, Neurosurgery and Psychiatry, vol. 79, no. 4, pp. 368-376, 2008.

[3] D. J. Gelb, E. Oliver, and S. Gilman, "Diagnostic criteria for Parkinson disease," Archives of Neurology, vol. 56, no. 1, pp. 33-39, 1999.

[4] E. Bezard, S. Dovero, C. Prunier et al., "Relationship between the appearance of symptoms and the level of nigrostriatal degeneration in a progressive 1-methyl-4-phenyl-1,2,3,6tetrahydropyridine-lesioned macaque model of Parkinson's disease," Journal of Neuroscience, vol. 21, no. 17, pp. 68536861, 2001.

[5] T. Wichmann and M. R. DeLong, "Functional neuroanatomy of the basal ganglia in Parkinson's disease," Advances in neurology, vol. 91, pp. 9-18, 2003.

[6] M. G. Spillantini, R. A. Crowther, R. Jakes, M. Hasegawa, and M. Goedert, “ $\alpha$-synuclein in filamentous inclusions of Lewy bodies from Parkinson's disease and dementia with Lewy bodies," Proceedings of the National Academy of Sciences of the United States of America, vol. 95, no. 11, pp. 6469-6473, 1998.

[7] J. L. Montastruc, O. Rascol, and J. M. Senard, "Current status of dopamine agonists in Parkinson's disease management," Drugs, vol. 46, no. 3, pp. 384-393, 1993.

[8] A. H. V. Schapira, "Molecular and clinical pathways to neuroprotection of dopaminergic drugs in Parkinson disease," Neurology, vol. 72, no. 7, pp. S44-S50, 2009.

[9] W. Dauer and S. Przedborski, "Parkinson's disease: mechanisms and models," Neuron, vol. 39, no. 6, pp. 889-909, 2003.

[10] S. Przedborski, V. Jackson-Lewis, M. Vila et al., "Free radical and nitric oxide toxicity in Parkinson's disease," Advances in neurology, vol. 91, pp. 83-94, 2003.

[11] G. W. Kreutzberg, "Microglia: a sensor for pathological events in the CNS," Trends in Neurosciences, vol. 19, no. 8, pp. 312-318, 1996.

[12] P. L. McGeer, S. Itagaki, B. E. Boyes, and E. G. McGeer, "Reactive microglia are positive for HLA-DR in the substantia nigra of Parkinson's and Alzheimer's disease brains," Neurology, vol. 38, no. 8, pp. 1285-1291, 1988.

[13] A. Nimmerjahn, F. Kirchhoff, and F. Helmchen, "Neuroscience: resting microglial cells are highly dynamic surveillants of brain parenchyma in vivo," Science, vol. 308, no. 5726, pp. 1314-1318, 2005.

[14] B. Liu and J. S. Hong, "Role of microglia in inflammationmediated neurodegenerative diseases: mechanisms and strategies for therapeutic intervention," Journal of Pharmacology and Experimental Therapeutics, vol. 304, no. 1, pp. 1-7, 2003.

[15] J. Gehrmann, Y. Matsumoto, and G. W. Kreutzberg, "Microglia: intrinsic immuneffector cell of the brain," Brain Research Reviews, vol. 20, no. 3, pp. 269-287, 1995.

[16] G. M. Hayes, M. N. Woodroofe, and M. L. Cuzner, "Microglia express MHC class II in normal and demyelinating human white matter," Annals of the New York Academy of Sciences, vol. 540, pp. 501-503, 1988.

[17] K. Imamura, N. Hishikawa, M. Sawada, T. Nagatsu, M. Yoshida, and Y. Hashizume, "Distribution of major histocompatibility complex class II-positive microglia and cytokine profile of Parkinson's disease brains," Acta Neuropathologica, vol. 106, no. 6, pp. 518-526, 2003. 
[18] W. F. Hickey and H. Kimura, "Perivascular microglial cells of the CNS are bone marrow-derived and present antigen in vivo," Science, vol. 239, no. 4837, pp. 290-292, 1988.

[19] M. L. Block, L. Zecca, and J. S. Hong, "Microgliamediated neurotoxicity: uncovering the molecular mechanisms," Nature Reviews Neuroscience, vol. 8, no. 1, pp. 57-69, 2007.

[20] W. J. Streit, S. A. Walter, and N. A. Pennell, "Reactive microgliosis," Progress in Neurobiology, vol. 57, no. 6, pp. 563-581, 1999.

[21] H. M. Gao and J. S. Hong, "Why neurodegenerative diseases are progressive: uncontrolled inflammation drives disease progression," Trends in Immunology, vol. 29, no. 8, pp. 357$365,2008$.

[22] A. Castaño, A. J. Herrera, J. Cano, and A. Machado, "Lipopolysaccharide intranigral injection induces inflammatory reaction and damage in nigrostriatal dopaminergic system," Journal of Neurochemistry, vol. 70, no. 4, pp. 15841592, 1998.

[23] H. M. Gao, J. Jiang, B. Wilson, W. Zhang, J. S. Hong, and B. Liu, "Microglial activation-mediated delayed and progressive degeneration of rat nigral dopaminergic neurons: relevance to Parkinson's disease," Journal of Neurochemistry, vol. 81, no. 6, pp. 1285-1297, 2002.

[24] H. M. Gao, B. Liu, W. Zhang, and J. S. Hong, "Critical role of microglial NADPH oxidase-derived free radicals in the in vitro MPTP model of Parkinson's disease," The FASEB Journal, vol. 17, pp. 1954-1956, 2003.

[25] M. Gerlach, P. Riederer, H. Przuntek, and M. B. H. Youdim, "MPTP mechanisms of neurotoxicity and their implications for Parkinson's disease," European Journal of Pharmacology, vol. 208, no. 4, pp. 273-286, 1991.

[26] D. C. Wu, V. Jackson-Lewis, M. Vila et al., "Blockade of microglial activation is neuroprotective in the 1-methyl-4phenyl-1,2,3,6-tetrahydropyridine mouse model of Parkinson disease," Journal of Neuroscience, vol. 22, no. 5, pp. 17631771, 2002.

[27] B. Liu, L. Du, and J. S. Hong, "Naloxone protects rat dopaminergic neurons against inflammatory damage through inhibition of microglia activation and superoxide generation," Journal of Pharmacology and Experimental Therapeutics, vol. 293, no. 2, pp. 607-617, 2000.

[28] H. Chen, S. M. Zhang, M. A. Hernán et al., "Nonsteroidal anti-inflammatory drugs and the risk of Parkinson disease," Archives of Neurology, vol. 60, no. 8, pp. 1059-1064, 2003.

[29] H. Chen, E. Jacobs, M. A. Schwarzschild et al., "Nonsteroidal antiinflammatory drug use and the risk for Parkinson's disease," Annals of Neurology, vol. 58, no. 6, pp. 963-967, 2005.

[30] H. Chen, E. J. O’Reilly, M. A. Schwarzschild, and A. Ascherio, "Peripheral inflammatory biomarkers and risk of Parkinson's disease," American Journal of Epidemiology, vol. 167, no. 1, pp. 90-95, 2008.

[31] A. Gerhard, N. Pavese, G. Hotton et al., "In vivo imaging of microglial activation with $\left[{ }^{11} \mathrm{C}\right](R)$-PK11195 PET in idiopathic Parkinson's disease," Neurobiology of Disease, vol. 21, no. 2, pp. 404-412, 2006.

[32] P. L. McGeer and E. G. McGeer, "Glial reactions in Parkinson's disease," Movement Disorders, vol. 23, no. 4, pp. 474483, 2008.

[33] P. L. McGeer, C. Schwab, A. Parent, and D. Doudet, "Presence of reactive microglia in monkey substantia nigra years after 1-methyl-4-phenyl-1,2,3,6-tetrahydropyridine administration," Annals of Neurology, vol. 54, no. 5, pp. 599-604, 2003.
[34] J. W. Langston, L. S. Forno, J. Tetrud, A. G. Reeves, J. A. Kaplan, and D. Karluk, "Evidence of active nerve cell degeneration in the substantia nigra of humans years after 1-methyl-4-phenyl-1,2,3,6-tetrahydropyridine exposure," Annals of Neurology, vol. 46, no. 4, pp. 598-605, 1999.

[35] C. Barcia, A. Sánchez Bahillo, E. Fernández-Villalba et al., "Evidence of active microglia in substantia nigra pars compacta of parkinsonian monkeys 1 year after MPTP exposure," GLIA, vol. 46, no. 4, pp. 402-409, 2004.

[36] Y. S. Kim, D. H. Choi, M. L. Block et al., "A pivotal role of matrix metalloproteinase-3 activity in dopaminergic neuronal degeneration via microglial activation," The FASEB Journal, vol. 21, no. 1, pp. 179-187, 2007.

[37] L. Zecca, F. A. Zucca, H. Wilms, and D. Sulzer, "Neuromelanin of the substantia nigra: a neuronal black hole with protective and toxic characteristics," Trends in Neurosciences, vol. 26, no. 11, pp. 578-580, 2003.

[38] W. Zhang, T. Wang, Z. Pei et al., "Aggregated $\alpha$-synuclein activates microglia: a process leading to disease progression in Parkinson's disease," The FASEB Journal, vol. 19, no. 6, pp. 533-542, 2005.

[39] H. M. Gao, P. T. Kotzbauer, K. Uryu, S. Leight, J. Q. Trojanowski, and V. M. Y. Lee, "Neuroinflammation and oxidation/nitration of $\alpha$-synuclein linked to dopaminergic neurodegeneration," Journal of Neuroscience, vol. 28, no. 30, pp. 7687-7698, 2008.

[40] Y. S. Kim and T. H. Joh, "Microglia, major player in the brain inflammation: their roles in the pathogenesis of Parkinson's disease," Experimental and Molecular Medicine, vol. 38, no. 4, pp. 333-347, 2006.

[41] C. C. Chao, S. Hu, T. W. Molitor, E. G. Shaskan, and P. K. Peterson, "Activated microglia mediate neuronal cell injury via a nitric oxide mechanism," Journal of Immunology, vol. 149, no. 8, pp. 2736-2741, 1992.

[42] P. Jenner, "Oxidative stress in Parkinson's disease," Annals of Neurology, vol. 53, no. 3, pp. S26-S38, 2003.

[43] E. Koutsilieri, C. Scheller, E. Grünblatt, K. Nara, J. Li, and P. Riederer, "Free radicals in Parkinson's disease," Journal of Neurology, vol. 249, supplement 2, pp. II1-II5, 2002.

[44] S. Jana, A. K. Maiti, M. B. Bagh et al., "Dopamine but not 3,4-dihydroxy phenylacetic acid (DOPAC) inhibits brain respiratory chain activity by autoxidation and mitochondria catalyzed oxidation to quinone products: implications in Parkinson's disease," Brain Research, vol. 1139, no. 1, pp. 195-200, 2007.

[45] D. T. Dexter, C. J. Carter, F. R. Wells et al., "Basal lipid peroxidation in substantia nigra is increased in Parkinson's disease," Journal of Neurochemistry, vol. 52, no. 2, pp. 381-389, 1989.

[46] R. N. Dilger and R. W. Johnson, "Aging, microglial cell priming, and the discordant central inflammatory response to signals from the peripheral immune system," Journal of Leukocyte Biology, vol. 84, no. 4, pp. 932-939, 2008.

[47] Y. Huang, C. J. Henry, R. Dantzer, R. W. Johnson, and J. P. Godbout, "Exaggerated sickness behavior and brain proinflammatory cytokine expression in aged mice in response to intracerebroventricular lipopolysaccharide," Neurobiology of Aging, vol. 29, no. 11, pp. 1744-1753, 2008.

[48] R. von Bernhardi, J. E. Tichauer, and J. Eugenín, "Agingdependent changes of microglial cells and their relevance for neurodegenerative disorders," Journal of Neurochemistry, vol. 112, no. 5, pp. 1099-1114, 2010.

[49] J. E. Merrill and E. N. Benveniste, "Cytokines in inflammatory brain lesions: helpful and harmful," Trends in Neurosciences, vol. 19, no. 8, pp. 331-338, 1996. 
[50] T. Nagatsu, M. Mogi, H. Ichinose, and A. Togari, "Cytokines in Parkinson's disease," Journal of Neural Transmission. Supplementa, no. 58, pp. 143-151, 2000.

[51] M. Mogi, M. Harada, P. Riederer, H. Narabayashi, K. Fujita, and T. Nagatsu, "Tumor necrosis factor- $\alpha$ (TNF- $\alpha$ ) increases both in the brain and in the cerebrospinal fluid from parkinsonian patients," Neuroscience Letters, vol. 165, no. 1-2, pp. 208-210, 1994.

[52] G. Stypuła, J. Kunert-Radek, H. Stepien, K. Zylińska, and M. Pawlikowski, "Evaluation of interleukins, ACTH, cortisol and prolactin concentrations in the blood of patients with Parkinson's disease," NeuroImmunoModulation, vol. 3, no. 2-3, pp. 131-134, 1996.

[53] E. C. Hirsch, "Glial cells and Parkinson's disease," Journal of Neurology, vol. 247, supplement 2, no. 2, pp. 58-62, 2000.

[54] T. Nagatsu and M. Sawada, "Inflammatory process in Parkinson's disease: role for cytokines," Current Pharmaceutical Design, vol. 11, no. 8, pp. 999-1016, 2005.

[55] P. Ravenscroft and J. Brotchie, "NMDA receptors in the basal ganglia," Journal of Anatomy, vol. 196, no. 4, pp. 577-585, 2000.

[56] E. A. Waxman and D. R. Lynch, "N-methyl-D-aspartate receptor subtype mediated bidirectional control of p38 mitogen-activated protein kinase," Journal of Biological Chemistry, vol. 280, no. 32, pp. 29322-29333, 2005.

[57] S. Kikuchi and S. U. Kim, "Glutamate neurotoxicity in mesencephalic dopaminergic neurons in culture," Journal of Neuroscience Research, vol. 36, no. 5, pp. 558-569, 1993.

[58] B. P. Connop, R. J. Boegman, K. Jhamandas, and R. J. Beninger, "Excitotoxic action of NMDA agonists on nigrostriatal dopaminergic neurons: modulation by inhibition of nitric oxide synthesis," Brain Research, vol. 676, no. 1, pp. 124-132, 1995.

[59] P. J. Hallett and D. G. Standaert, "Rationale for and use of NMDA receptor antagonists in Parkinson's disease," Pharmacology \& Therapeutics, vol. 102, no. 2, pp. 155-174, 2004.

[60] W. J. Schmidt, M. Bubser, and W. Hauber, "Behavioural pharmacology of glutamate in the basal ganglia," Journal of Neural Transmission. Supplementa, no. 38, pp. 65-89, 1992.

[61] H. Takeuchi, T. Mizuno, G. Zhang et al., "Neuritic beading induced by activated microglia is an early feature of neuronal dysfunction toward neuronal death by inhibition of mitochondrial respiration and axonal transport," Journal of Biological Chemistry, vol. 280, no. 11, pp. 10444-10454, 2005.

[62] P. M. Mattila, J. O. Rinne, H. Helenius, and M. Röyttä, "Neuritic degeneration in the hippocampus and amygdala in Parkinson's disease in relation to Alzheimer pathology," Acta Neuropathologica, vol. 98, no. 2, pp. 157-164, 1999.

[63] C. Ikonomidou and L. Turski, "Neurodegenerative disorders: clues from glutamate and energy metabolism," Critical Reviews in Neurobiology, vol. 10, no. 2, pp. 239-263, 1996.

[64] J. T. Greenamyre and C. F. O'Brien, "N-methyl-D-aspartate antagonists in the treatment of Parkinson's disease," Archives of Neurology, vol. 48, no. 9, pp. 977-981, 1991.

[65] D. Aarsland, C. Ballard, Z. Walker et al., "Memantine in patients with Parkinson's disease dementia or dementia with Lewy bodies: a double-blind, placebo-controlled, multicentre trial," The Lancet Neurology, vol. 8, no. 7, pp. 613-618, 2009.

[66] L. Verhagen Metman, P. Del Dotto, P. van den Munckhof, J. Fang, M. M. Mouradian, and T. N. Chase, "Amantadine as treatment for dyskinesias and motor fluctuations in Parkinson's disease," Neurology, vol. 50, no. 5, pp. 1323-1326, 1998.
[67] J. R. Moffett and M. A. Namboodiri, "Tryptophan and the immune response," Immunology and Cell Biology, vol. 81, no. 4, pp. 247-265, 2003.

[68] A. L. Mellor, B. Baban, P. Chandler et al., "Cutting edge: induced indoleamine 2,3 dioxygenase expression in dendritic cell subsets suppresses T cell clonal expansion," Journal of Immunology, vol. 171, no. 4, pp. 1652-1655, 2003.

[69] D. H. Munn and A. L. Mellor, "IDO and tolerance to tumors," Trends in Molecular Medicine, vol. 10, no. 1, pp. 15-18, 2004.

[70] G. J. Guillemin, D. G. Smith, G. A. Smythe, P. J. Armati, and B. J. Brew, "Expression of the kynurenine pathway enzymes in human microglia and macrophages," Advances in Experimental Medicine and Biology, vol. 527, pp. 105-112, 2003.

[71] G. J. Guillemin, S. J. Kerr, G. A. Smythe et al., "Kynurenine pathway metabolism in human astrocytes: a paradox for neuronal protection," Journal of Neurochemistry, vol. 78, no. 4, pp. 842-853, 2001.

[72] G. J. Guillemin, K. M. Cullen, C. K. Lim et al., "Characterization of the kynurenine pathway in human neurons," Journal of Neuroscience, vol. 27, no. 47, pp. 12884-12892, 2007.

[73] R. Owe-Young, N. L. Webster, M. Mukhtar et al., "Kynurenine pathway metabolism in human blood-brain-barrier cells: implications for immune tolerance and neurotoxicity," Journal of Neurochemistry, vol. 105, no. 4, pp. 1346-1357, 2008.

[74] H. Q. Wu, P. Guidetti, J. H. Goodman et al., "Kynurenergic manipulations influence excitatory synaptic function and excitotoxic vulnerability in the rat hippocampus in vivo," Neuroscience, vol. 97, no. 2, pp. 243-251, 2000.

[75] A. Chiarugi, E. Meli, and F. Moroni, "Similarities and differences in the neuronal death processes activated by $3 \mathrm{OH}-\mathrm{kynurenine}$ and quinolinic acid," Journal of Neurochemistry, vol. 77, no. 5, pp. 1310-1318, 2001.

[76] R. Schwarcz and R. Pellicciari, "Manipulation of brain kynurenines: glial targets, neuronal effects, and clinical opportunities," Journal of Pharmacology and Experimental Therapeutics, vol. 303, no. 1, pp. 1-10, 2002.

[77] A. J. Smith, T. W. Stone, and R. A. Smith, "Neurotoxicity of tryptophan metabolites," Biochemical Society Transactions, vol. 35, no. 5, pp. 1287-1289, 2007.

[78] A. J. Smith, R. A. Smith, and T. W. Stone, "5-hydroxyanthranilic acid, a tryptophan metabolite, generates oxidative stress and neuronal death via p38 activation in cultured cerebellar granule neurones," Neurotoxicity Re-search, vol. 15, no. 4, pp. 303-310, 2009.

[79] S. Okuda, N. Nishiyama, H. Saito, and H. Katsuki, "3-hydroxykynurenine, an endogenous oxidative stress generator, causes neuronal cell death with apoptotic features and region selectivity," Journal of Neurochemistry, vol. 70, no. 1, pp. 299-307, 1998.

[80] T. W. Stone and M. N. Perkins, "Quinolinic acid: a potent endogenous excitant at amino acid receptors in CNS," European Journal of Pharmacology, vol. 72, no. 4, pp. 411-412, 1981.

[81] R. Schwarcz, W. O. Whetsell, and R. M. Mangano, "Quinolinic acid: an endogenous metabolite that produces axonsparing lesions in rat brain," Science, vol. 219, no. 4582, pp. 316-318, 1983.

[82] G. J. Guillemin and B. J. Brew, "Implications of the kynurenine pathway and quinolinic acid in Alzheimer's disease," Redox Report, vol. 7, no. 4, pp. 199-206, 2002. 
[83] G. J. Guillemin, L. Wang, and B. J. Brew, "Quinolinic acid selectively induces apoptosis of human astocytes: potential role in AIDS dementia complex," Journal of Neuroinflammation, vol. 2, article 16, 2005.

[84] A. Schurr, C. A. West, and B. M. Rigor, "Neurotoxicity of quinolinic acid and its derivatives in hypoxic rat hippocampal slices," Brain Research, vol. 568, no. 1-2, pp. 199-204, 1991.

[85] Y. M. Bordelon, M. F. Chesselet, D. Nelson, F. Welsh, and M. Erecińska, "Energetic dysfunction in quinolinic acid-lesioned rat striatum," Journal of Neurochemistry, vol. 69, no. 4, pp. 1629-1639, 1997.

[86] H. Baran, B. Kepplinger, M. Herrera-Marschitz, K. Stolze, G. Lubec, and H. Nohl, "Increased kynurenic acid in the brain after neonatal asphyxia," Life Sciences, vol. 69, no. 11, pp. 1249-1256, 2001.

[87] N. Braidy, R. Grant, S. Adams, B. J. Brew, and G. J. Guillemin, "Mechanism for quinolinic acid cytotoxicity in human astrocytes and neurons," Neurotoxicity Research, vol. 16, no. 1, pp. 77-86, 2009.

[88] G. J. Guillemin, K. M. Cullen, C. K. Lim et al., "Characterization of the kynurenine pathway in human neurons," Journal of Neuroscience, vol. 27, no. 47, pp. 12884-12892, 2007.

[89] G. J. Guillemin, G. Smythe, O. Takikawa, and B. J. Brew, "Expression of indoleamine 2,3-dioxygenase and production of quinolinic acid by human microglia, astrocytes, and neurons," GLIA, vol. 49, no. 1, pp. 15-23, 2005.

[90] P. Guidetti and R. Schwarcz, "3-hydroxykynurenine potentiates quinolinate but not NMDA toxicity in the rat striatum," European Journal of Neuroscience, vol. 11, no. 11, pp. 3857-3863, 1999.

[91] W. M. H. Behan and T. W. Stone, "Enhanced neuronal damage by co-administration of quinolinic acid and free radicals, and protection by adenosine $\mathrm{A}_{2 \mathrm{~A}}$ receptor antagonists," British Journal of Pharmacology, vol. 135, no. 6, pp. 1435-1442, 2002.

[92] I. Ghorayeb, Z. Puschban, P. O. Fernagut et al., "Simultaneous intrastriatal 6-hydroxydopamine and quinolinic acid injection: a model of early-stage striatonigral degeneration," Experimental Neurology, vol. 167, no. 1, pp. 133-147, 2001.

[93] M. G. Espey, O. N. Chernyshev, J. F. Reinhard, M. A. A. Namboodiri, and C. A. Colton, "Activated human microglia produce the excitotoxin quinolinic acid," NeuroReport, vol. 8, no. 2, pp. 431-434, 1997.

[94] M. P. Heyes, K. Saito, and S. P. Markey, "Human macrophages convert L-tryptophan into the neurotoxin quinolinic acid," Biochemical Journal, vol. 283, no. 3, pp. 633-635, 1992.

[95] H. E. Scharfman, P. S. Hodgkins, S. C. Lee, and R. Schwarcz, "Quantitative differences in the effects of de novo produced and exogenous kynurenic acid in rat brain slices," Neuroscience Letters, vol. 274, no. 2, pp. 111-114, 1999.

[96] A. F. Miranda, R. J. Boegman, R. J. Beninger, and K. Jhamandas, "Protection against quinolinic acid-mediated excitotoxicity in nigrostriatal dopaminergic neurons by endogenous kynurenic acid," Neuroscience, vol. 78, no. 4, pp. 967-975, 1997.

[97] R. Carpenedo, A. Pittaluga, A. Cozzi et al., "Presynaptic kynurenate-sensitive receptors inhibit glutamate release," European Journal of Neuroscience, vol. 13, no. 11, pp. 2141-2147, 2001.

[98] R. C. Roberts, K. E. McCarthy, F. Du, E. Okuno, and R. Schwarcz, "Immunocytochemical localization of the quinolinic acid synthesizing enzyme, 3-hydroxyanthranilic acid oxygenase, in the rat substantia nigra," Brain Research, vol. 650, no. 2, pp. 229-238, 1994.
[99] R. Schwarcz, F. Du, W. Schmidt et al., "Kynurenic acid: a potential pathogen in brain disorders," Annals of the New York Academy of Sciences, vol. 648, pp. 140-153, 1992.

[100] A. C. Foster, A. Vezzani, E. D. French, and R. Schwarcz, "Kynurenic acid blocks neurotoxicity and seizures induced in rats by the related brain metabolite quinolinic acid," Neuroscience Letters, vol. 48, no. 3, pp. 273-278, 1984.

[101] K. Jhamandas, R. J. Boegman, R. J. Beninger, and M. Bialik, "Quinolinate-induced cortical cholinergic damage: modulation by tryptophan metabolites," Brain Research, vol. 529, no. 1-2, pp. 185-191, 1990.

[102] U. Testa, F. Louache, M. Titeux, P. Thomopoulos, and H. Rochant, "The iron-chelating agent picolinic acid enhances transferrin receptors expression in human erythroleukaemic cell lines," British Journal of Haematology, vol. 60, no. 3, pp. 491-502, 1985.

[103] F. Molina-Holgado, R. C. Hider, A. Gaeta, R. Williams, and P. Francis, "Metals ions and neurodegeneration," BioMetals, vol. 20, no. 3-4, pp. 639-654, 2007.

[104] G. J. Guillemin, S. J. Kerr, L. A. Pemberton et al., "IFN$\beta$ induces kynurenine pathway metabolism in human macrophages: potential implications for multiple sclerosis treatment," Journal of Interferon and Cytokine Research, vol. 21, no. 12, pp. 1097-1101, 2001.

[105] R. J. Beninger, A. M. Colton, J. L. Ingles, K. Jhamandas, and R. J. Boegman, "Picolinic acid blocks the neurotoxic but not the neuroexcitant properties of quinolinic acid in the rat brain: evidence from turning behaviour and tyrosine hydroxylase immunohistochemistry," Neuroscience, vol. 61, no. 3, pp. 603-612, 1994.

[106] R. Owe-Young, N. L. Webster, M. Mukhtar et al., "Kynurenine pathway metabolism in human blood-brain-barrier cells: implications for immune tolerance and neurotoxicity," Journal of Neurochemistry, vol. 105, no. 4, pp. 1346-1357, 2008.

[107] T. Ogawa, W. R. Matson, M. F. Beal et al., "Kynurenine pathway abnormalities in Parkinson's disease," Neurology, vol. 42, no. 9, pp. 1702-1706, 1992.

[108] M. Flint Beal, W. R. Matson, E. Storey et al., "Kynurenic acid concentrations are reduced in Huntington's disease cerebral cortex," Journal of the Neurological Sciences, vol. 108, no. 1, pp. 80-87, 1992.

[109] E. Knyihár-Csillik, B. Csillik, M. Pákáski et al., "Decreased expression of kynurenine aminotransferase-I (KAT-I) in the substantia nigra of mice after 1-methyl-4-phenyl-1,2,3,6tetrahydropyridine (MPTP) treatment," Neuroscience, vol. 126, no. 4, pp. 899-914, 2004.

[110] M. Merino, M. L. Vizuete, J. Cano, and A. Machado, "The non-NMDA glutamate receptor antagonists 6-cyano-7nitroquinoxaline-2,3-dione and 2,3-dihydroxy-6-nitro-7sulfamoylbenzo $(f)$ quinoxaline, but not NMDA antagonists, block the intrastriatal neurotoxic effect of $\mathrm{MPP}^{+}$," Journal of Neurochemistry, vol. 73, no. 2, pp. 750-757, 1999.

[111] Z. Hartai, P. Klivenyi, T. Janaky, B. Penke, L. Dux, and L. Vecsei, "Kynurenine metabolism in plasma and in red blood cells in Parkinson's disease," Journal of the Neurological Sciences, vol. 239, no. 1, pp. 31-35, 2005.

[112] S. Fukui, R. Schwarcz, S. I. Rapoport, Y. Takada, and Q. R. Smith, "Blood-brain barrier transport of kynurenines: implications for brain synthesis and metabolism," Journal of Neurochemistry, vol. 56, no. 6, pp. 2007-2017, 1991.

[113] M. C. Barth, N. Ahluwalia, T. J. T. Anderson et al., "Kynurenic acid triggers firm arrest of leukocytes to vascular 
endothelium under flow conditions," Journal of Biological Chemistry, vol. 284, no. 29, pp. 19189-19195, 2009.

[114] H. Németh, J. Toldi, and L. Vécsei, "Kynurenines, Parkinson's disease and other neurodegenerative disorders: preclinical and clinical studies," Journal of Neural Transmission. Supplementa, no. 70, pp. 285-304, 2006.

[115] S. Fujigaki, K. Saito, K. Sekikawa et al., "Lipopolysaccharide induction of indoleamine 2,3-dioxygenase is mediated dominantly by an IFN- $\boldsymbol{\gamma}$-independent mechanism," European Journal of Immunology, vol. 31, no. 8, pp. 2313-2318, 2001.

[116] T. W. Stone and L. G. Darlington, "Endogenous kynurenines as targets for drug discovery and development," Nature Reviews Drug Discovery, vol. 1, no. 8, pp. 609-620, 2002.

[117] L. McNally, Z. Bhagwagar, and J. Hannestad, "Inflammation, glutamate, and glia in depression: a literature review," CNS Spectrums, vol. 13, no. 6, pp. 501-510, 2008.

[118] H. Q. Wu, S. C. Lee, and R. Schwarcz, "Systemic administration of 4-chlorokynurenine prevents quinolinate neurotoxicity in the rat hippocampus," European Journal of Pharmacology, vol. 390, no. 3, pp. 267-274, 2000.

[119] T. W. Stone, "Development and therapeutic potential of kynurenic acid and kynurenine derivatives for neuroprotection," Trends in Pharmacological Sciences, vol. 21, no. 4, pp. 149-154, 2000.

[120] M. Platten, P. P. Ho, and L. Steinman, "Anti-inflammatory strategies for the treatment of multiple sclerosistryptophan catabolites may hold the key," Drug Discovery Today: Therapeutic Strategies, vol. 3, no. 3, pp. 401-408, 2006.

[121] M. Platten, P. P. Ho, S. Youssef et al., "Treatment of autoimmune neuroinflammation with a synthetic tryptophan metabolite," Science, vol. 310, no. 5749, pp. 850-855, 2005.

[122] C. W. Ritchie, A. I. Bush, and C. L. Masters, "Metal-protein attenuating compounds and Alzheimer's disease," Expert Opinion on Investigational Drugs, vol. 13, no. 12, pp. 1585-1592, 2004.

[123] J. R. Moffett, T. Els, M. G. Espey, S. A. Walter, W. J. Streit, and M. A. A. Namboodiri, "Quinolinate immunoreactivity in experimental rat brain tumors is present in macrophages but not in astrocytes," Experimental Neurology, vol. 144, no. 2, pp. 287-301, 1997.

[124] M. Hamann, S. E. Sander, and A. Richter, "Effects of the kynurenine 3-hydroxylase inhibitor Ro 61-8048 after intrastriatal injections on the severity of dystonia in the $\mathrm{dt}$ mutant," European Journal of Pharmacology, vol. 586, no. 1-3, pp. 156-159, 2008. 


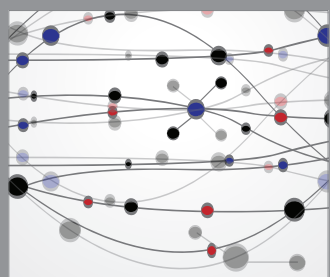

The Scientific World Journal
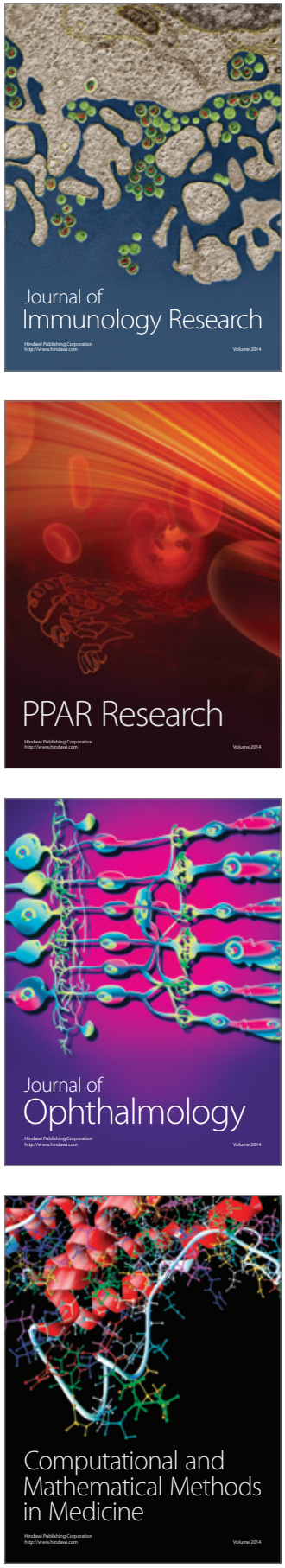

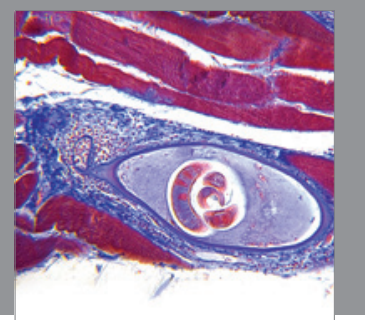

Gastroenterology

Research and Practice
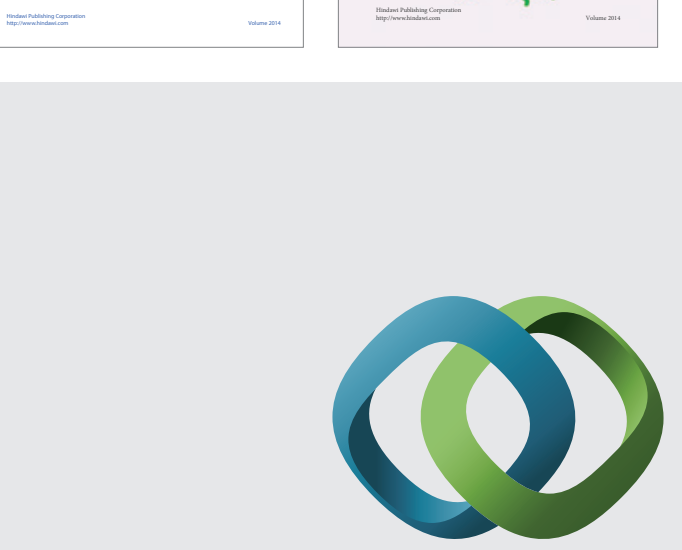

\section{Hindawi}

Submit your manuscripts at

http://www.hindawi.com
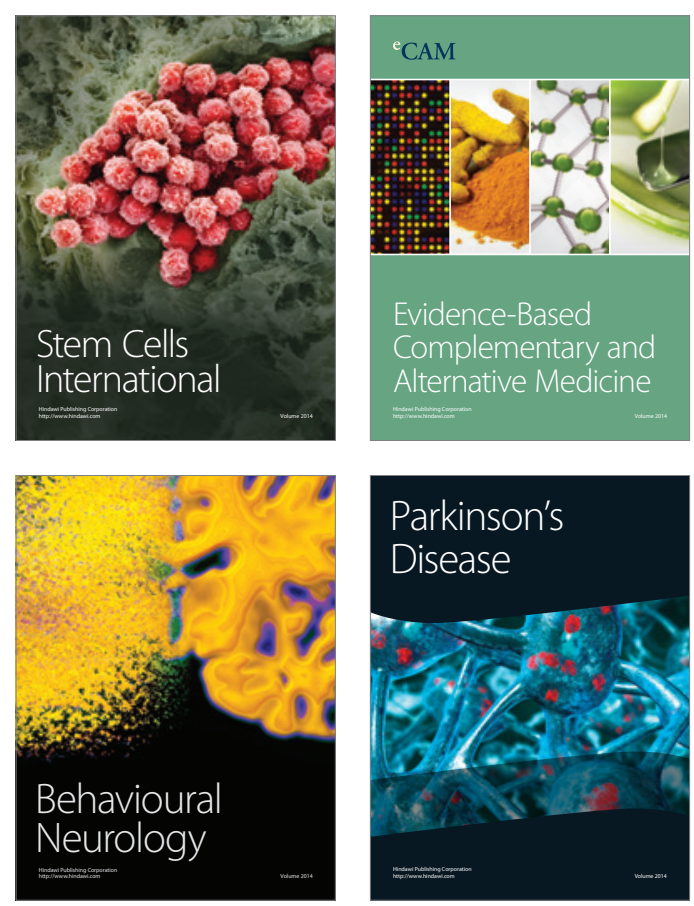

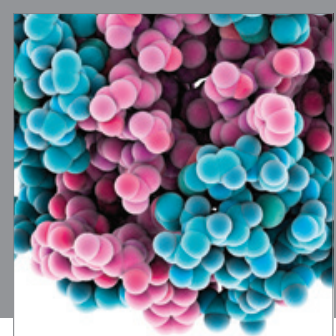

Journal of
Diabetes Research

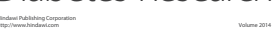

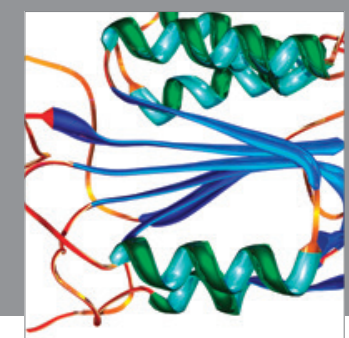

Disease Markers
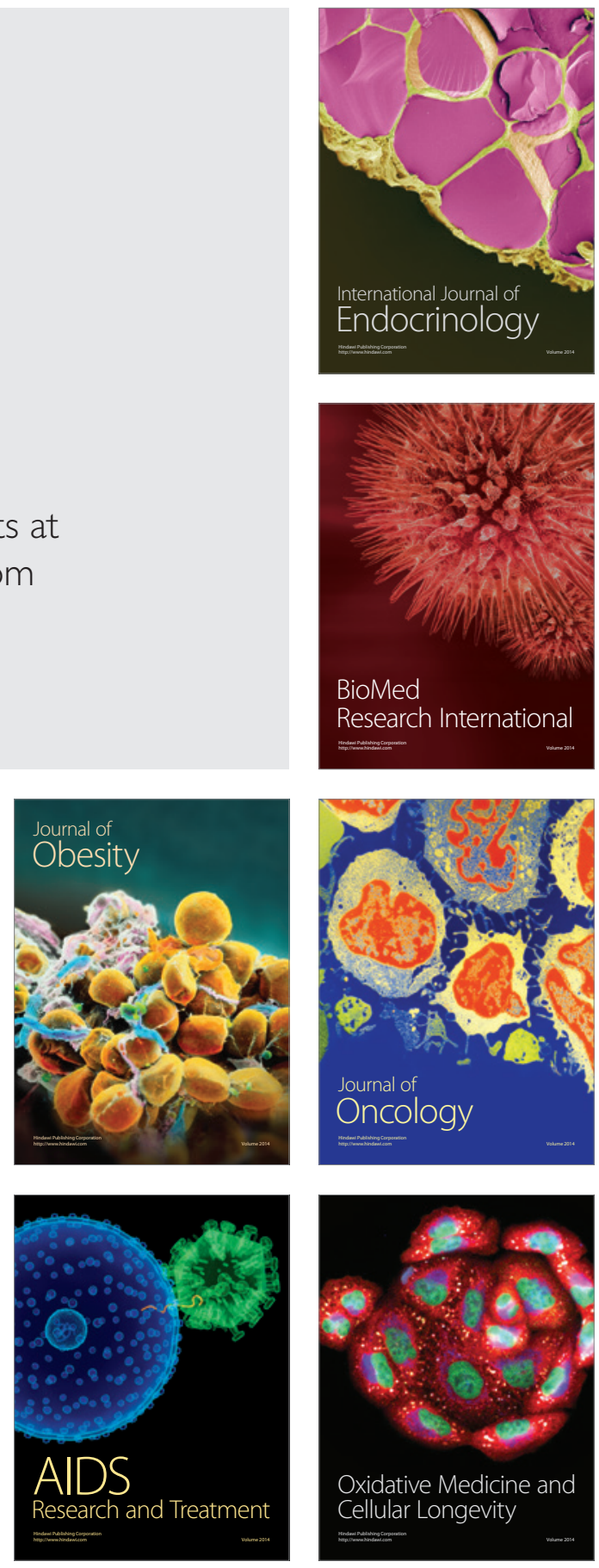